\title{
PREVALENCIA DE INFECCIONES RESPIRATORIAS Y ENFERMEDADES DIARREICAS AGUDAS EN NIÑOS DE VILLA CARMEN - QUILLACOLLO, 2017
}

'Médico Cirujano. Hospital "Dr. Benigno Sánchez" - Quillacollo (Cochabamba - Bolivia).

Palabras clave: Enfermedades respiratorias, diarrea, prevalencia, niño.

Keywords: Respiratory Tract diseases, diarrhea, prevalence, child.

Correspondencia a: Dr. Alfredo Sejas Claros: al-sejas@hotmail.com

Recibido para publicación: 29 de abril 2018 Aceptado para publicación: 14 de julio 2018

Citar como: Rev Cient Cienc Med 2018;20(1):50-59

\section{PREVALENCE OF RESPIRATORY INFECTIONS AND ACUTE DIARRHEAL DISEASES IN CHILDREN OF VILLA CARMEN - QUILLACOLLO, 2017}

Dr. Alfredo Sejas Claros ${ }^{1}$, Dra. Rocio Condori Bustillos ${ }^{1}$

\section{RESUMEN}

Introducción: Las infecciones respiratorias y enfermedades diarreicas agudas son enfermedades prevalentes en el menor de 5 años, factores socioeconómicos bajos y una deficiente higiene favorecen el desarrollo de estas patologías. Objetivo: Determinar la prevalencia de estas enfermedades en el menor de 5 años en la comunidad de Villa Carmen - Quillacollo durante el año 2017. Materiales y Métodos: Se realizó un estudio descriptivo, de corte transversal. El universo fue de 1780 menores de 5 años que acudieron a consulta del centro de salud de Villa Carmen - Quillacollo. Resultados: Se encontró que $65 \mathrm{I}$ menores de 5 años enfermaron con infecciones respiratorias (37\%) y 222 enfermaron con enfermedades diarreicas agudas (12\%). La mayor prevalencia de infecciones respiratorias fue en los meses de abril, mayo, julio y la mayor prevalencia de enfermedades diarreicas fue en los meses de marzo, abril, junio, julio y diciembre. Se identificó que los niños de I año a menores de 2 años son los más afectados con infecciones respiratorias presentando resfrió común y los menores de I año son los más afectados con enfermedades diarreicas presentando diarrea y diarrea persistente. Conclusión: La prevalencia de infecciones respiratorias y enfermedades diarreicas agudas en la comunidad de Villa Carmen es de $37 \%$ y $12 \%$ respectivamente, datos que tienen que ser puestos a consideración, por lo cual se recomienda información y educación a las madres y niños respecto a medidas preventivas para limitar la prevalencia de estas patologías.

\section{ABSTRACT}

Introduction: Respiratory infections and acute diarrheal diseases are diseases prevalent in children under 5 years old, low socioeconomic factors and poor hygiene favor the development of these pathologies. Objective: Determine the prevalence of these diseases in children under 5 years old in the community of Villa Carmen - Quillacollo during 2017. Materials and Methods:A descriptive, cross-sectional study was carried out. The universe was of I 780 children under 5 years of age who visited the health center of Villa Carmen - Quillacollo. Results: It was found that $65 \mathrm{I}$ children under 5 years of age became ill with respiratory infections (37\%) and 222 became ill with acute diarrheal diseases (12\%). The highest prevalence of respiratory infections was in the months of April, May, July and the highest prevalence of diarrheal diseases was in the months of March, April, June, July and December. It was identified that children aged I year under 2 are the most affected with respiratory infections presenting common cold and children under $I$ are the most affected with diarrheal diseases presenting diarrhea and persistent diarrhea. Conclusion:The prevalence of respiratory infections and acute diarrheal diseases in the community of Villa Carmen is $37 \%$ and $12 \%$ respectively, data that have to be considered, for which information and education is recommended to mothers and children regarding preventive measures to limit the prevalence of these pathologies. 
INTRODUCCIÓN

Las as Infecciones Respiratorias Agudas (IRAS) y las Enfermeda_des Diarreicas Agudas (EDAS) componen a nivel mundial y sobretodo en países subdesarrollados, las causas principales de morbimortalidad infantil, trascendentalmente en el menor de 5 años. En nuestro país la mortalidad infantil tiene como causantes principales a estas dos patologías ${ }^{1,2}$.

Las IRAS y EDAS son las infecciones que más se relacionan con el menor de 5 años y las que se relacionan principalmente con una deficiente higiene, factores socioeconómicos desventajosos (pobreza, baja escolaridad de padres o tutores, inasistencia a controles del niño en centros de salud, edad materna) y la falta de conocimiento de los padres sobre los signos de alarma de estas enfermedades ${ }^{3}$.

Las IRAS se definen como la agrupación de enfermedades del aparato respiratorio transmisibles (resfrío común, sinusitis, otitis, faringitis aguda, faringoamigdalitis, laringitis, bronquitis, bronquiolitis, laringotraqueítis, hasta llegar a la neumonía), con los siguientes síntomas característicos: obstrucción nasal, tos, otalgia, rinorrea, odinofagia, dificultad respiratoria o respiración ruidosa y disfonía, la fiebre puede acompañar a este cuadro clínico. Mientras que la diarrea aguda se define como la presencia de heces acuosas o líquidas con una frecuencia de deposiciones mayor a tres veces en 24 horas, puede acompañarse de dolor abdominal, síntomas de deshidratación, náuseas, vómitos y dependiendo del origen puede presentarse fiebre o deposiciones con sangre. Los agentes causantes de diarrea son los virus (diarrea por rotavirus), bacterias (gastroenteritis, diarrea infecciosa por enterobacterias) y los parásitos (amebiasis, giardiasis). La principal complicación de EDAS es la deshidratación, mientras que la neumonía es la principal complicación de IRAS, ambas complicaciones son las causantes de las muertes en el menor de 5 años cuando estas infecciones no son tratadas en su debido tiempo ${ }^{4,5}$.

La comunidad de Villa Carmen ubicada en el municipio de Quillacollo, es un área rural - urbana dedicada a la agricultura, presentando familias humildes y emergentes con un crecimiento demográfico. No existen estudios reportados sobre la prevalencia de IRAS y EDAS sobre esta población, por lo cual el presente trabajo pretende determinar la prevalencia de IRAS y EDAS en el menor de 5 años en la comunidad de Villa Carmen Quillacollo durante el año 2017.

\section{MATERIALES Y MÉTODOS}

El presente trabajo es de tipo descriptivo y de corte transversal. El universo son todos los menores de 5 años que asistieron al centro de salud de la comunidad de Villa Carmen del municipio de Quillacollo, haciendo un total de 1780 niños, durante los meses de enero a diciembre del año 2017.

La muestra está comprendida por 873 casos de menores de 5 años que presentaron IRAS y EDAS. Seleccionados mediante un muestreo de tipo no probabilístico. Cabe mencionar que la cantidad restante de 907 niños menores de 5 años fueron atendidos por otras patologías (dermatitis de contacto, con- juntivitis bacteriana, varicela, sarcoptosis, dermatitis del pañal, micosis cutánea, impétigo, piodermitis entre otros) y atendidos para control de niño sano.

Criterios de inclusión: Todos los menores de 5 años que enfermaron con EDAS e IRAS en la comunidad de Villa Carmen Quillacollo, atendidos en el centro de salud de la comunidad, durante el año 2017.

Criterios de exclusión: Menores de 5 años con IRAS y EDAS atendidos en el centro de salud de Villa Carmen que no viven en la comunidad de Villa Carmen.

Las variables: Prevalencia global, por meses y por rango de edad más afectado.

En el presente estudio se utilizaron: Plantillas de recolección y procesamiento de datos y plantillas para el registro de resultados.

Se realizó una revisión exhaustiva de todas las historias clínicas de los menores de 5 años, revisión de registros de atención al menor de 5 años del Sistema Nacional de Información en Salud (SNIS) y revisión de fichas epidemiológicas del centro de salud de Villa Carmen del municipio de Quillacollo, con la finalidad de cuantificar a los pacientes menores de 5 años que presentaron IRAS y EDAS, atendidos de enero a diciembre de 2017. Se procedió con la cuantificación de los menores de 5 años con IRAS, registrando las siguientes patologías: resfrío común, faringitis agua, faringoamigdalitis y neumonía no grave. Y la cuantificación de los menores de 5 años con EDAS, registrando lo siguiente: amebiasis, giardiasis, gastroenteritis bacteriana, diarrea y diarrea persistente. Se procesaron los datos para obtener los porcentajes mensuales y para determinar la prevalencia global de ambas patologías. También se realizó una cuantificación de acuerdo a la edad (menor de 1 año, 1 año a menor de 2 años, 2 años a menor de 5 años), para determinar el grupo de edad más afectado por estas enfermedades.

Los resultados obtenidos fueron tabulados y procesados en gráficos en los programas de Microsoft Office Word y Excel $2010^{\circledR}$.

Limitaciones: No se podrá conocer si algunos menores de 5 años de la comunidad de Villa Carmen con IRAS o EDAS fueron atendidos en otros centros de salud como también si presentaron complicaciones, tampoco el número de menores de 5 años con estas patologías que no fueron llevados a consulta médica por sus padres.

Consideraciones éticas: Ya que se utilizaron historias clínicas de pacientes y registros del centro de salud de Villa Carmen, se solicitó el permiso pertinente del centro de salud para la utilización de los datos.

\section{RESULTADOS}

En el transcurso de enero a diciembre del año 2017 se atendió un total de 1780 menores de 5 años de los cuales 651 corresponden a niños enfermos con IRAS (37\%) y 222 corresponden a niños enfermos con EDAS (12\%). (Ver gráfico 1). 
Se encontró la mayor prevalencia de IRAS en los meses de abril, mayo, julio y la mayor prevalencia de EDAS en los meses de marzo, abril, junio, julio y diciembre. (Ver tabla 1 y gráfico 2). Se identificó que los niños de 1 año a menores de
2 años son los más afectados con IRAS presentando resfrío común. (Ver tabla 2) Respecto a EDAS se identificó que los menores de 1 año son los más afectados presentando diarrea y diarrea persistente. (Ver tabla 3)
Gráfico 1. Prevalencia global de IRAS y EDAS en el menor de 5 años

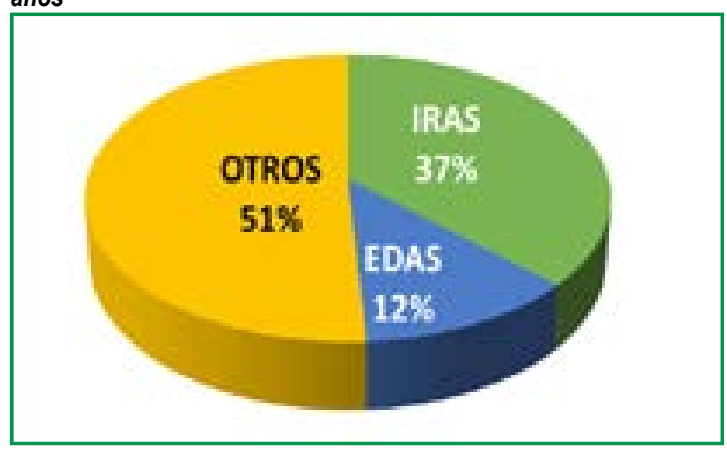

Grafico 2. Picos de prevalencia de IRAS y EDAS durante el 2017 en el menor de 5 años

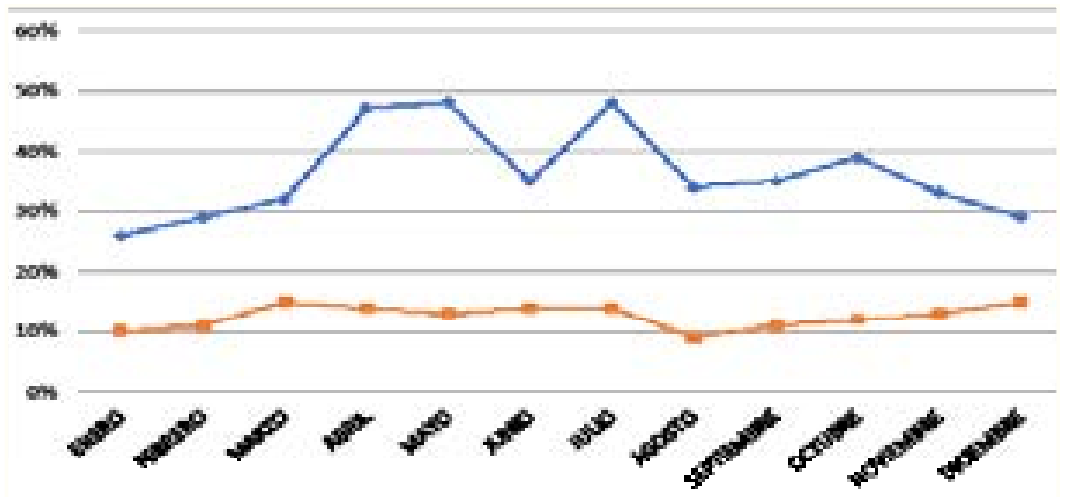

Tabla 1. Prevalencia de IRAS y EDAS por mes en el menor de 5 años

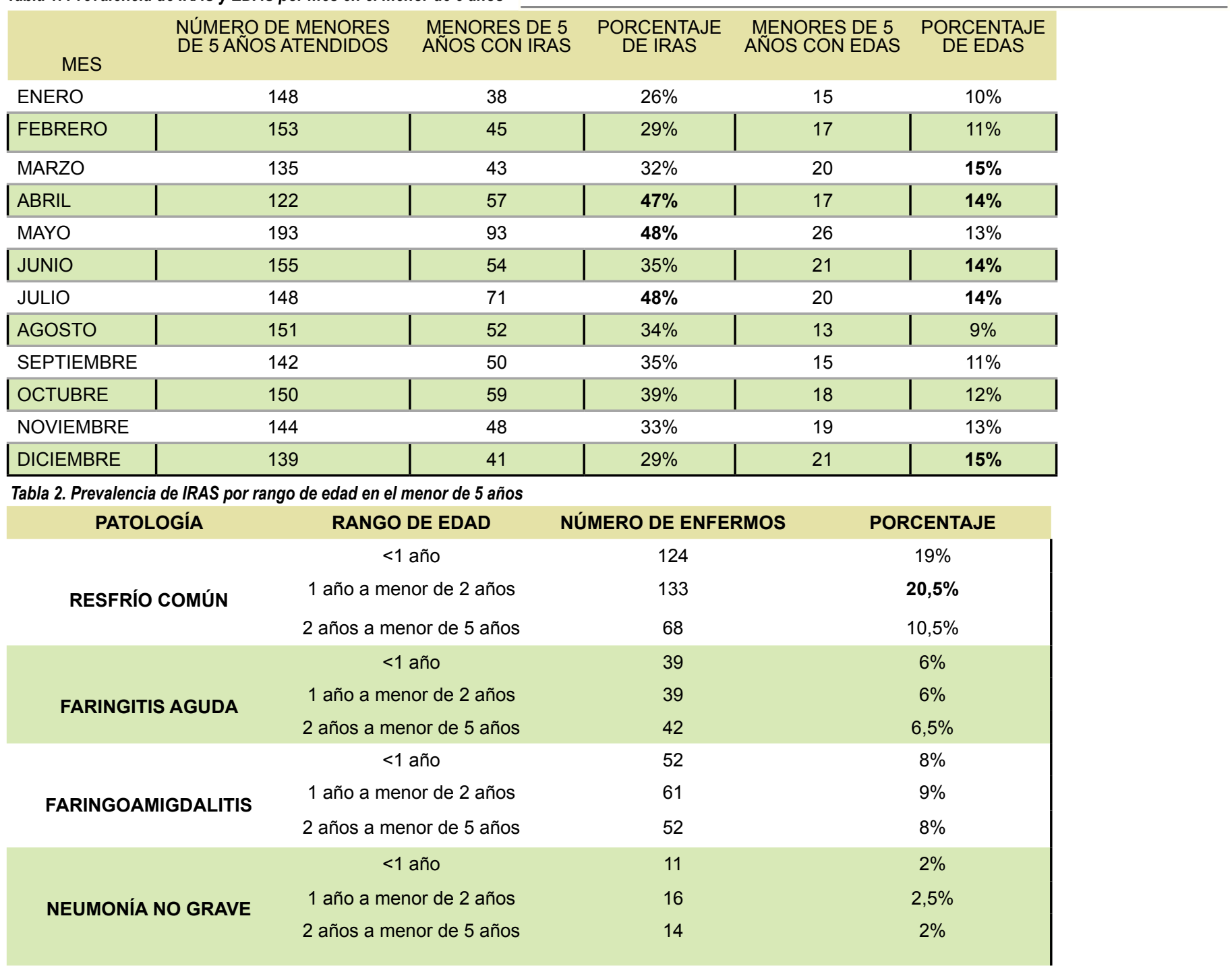


Tabla 3. Prevalencia de EDAS por rango de edad en el menor de 5 años

\begin{tabular}{|c|c|c|c|}
\hline PATOLOGIA & RANGO DE EDAD & NÚMERO DE ENFERMOS & PORCENTAJE \\
\hline \multirow{3}{*}{$\begin{array}{l}\text { DIARREA Y DIARREA } \\
\text { PERSISTENTE }\end{array}$} & $<1$ año & 67 & $30 \%$ \\
\hline & 1 año a menor de 2 años & 24 & $11 \%$ \\
\hline & 2 años a menor de 5 años & 9 & $4 \%$ \\
\hline \multirow{3}{*}{ AMEBIASIS } & $<1$ año & 14 & $6 \%$ \\
\hline & 1 año a menor de 2 años & 17 & $7,5 \%$ \\
\hline & 2 años a menor de 5 años & 19 & $8,5 \%$ \\
\hline \multirow{3}{*}{ GIARDIASIS } & $<1$ año & 6 & $3 \%$ \\
\hline & 1 año a menor de 2 años & 9 & $4 \%$ \\
\hline & 2 años a menor de 5 años & 11 & $5 \%$ \\
\hline \multirow{3}{*}{$\begin{array}{l}\text { GASTROENTERITIS } \\
\text { BACTERIANA }\end{array}$} & $<1$ año & 9 & $4 \%$ \\
\hline & 1 año a menor de 2 años & 13 & $6 \%$ \\
\hline & 2 años a menor de 5 años & 24 & $11 \%$ \\
\hline
\end{tabular}

\section{DISCUSIÓN}

La determinación de la prevalencia de IRAS y EDAS en el presente estudio nos abre una puerta a un conjunto de posibilidades y riesgos que están presentes y son comentados ampliamente en la literatura; tales como la mayor o menor prevalencia en la población infantil, sobretodo en el menor de 5 años y las muy comentadas complicaciones de estas patologías como causas de mortalidad infantil. Otro aspecto es la facilidad de la diseminación de estas patologías ya sea de persona a persona, a través de fómites o factores ambientales (contaminación atmosférica) en el caso de IRAS, sobre todo en los niños y la contaminación fecal en el caso de EDAS, muy frecuente en nuestro medio, encontrándose alimentos contaminados los cuales mediante una mala higiene contribuyen al desarrollo de la enfermedad ${ }^{6,7}$.

Este estudio determinó la prevalencia de IRAS y EDAS en el menor de 5 años en la comunidad de Villa Carmen ubicado en la provincia de Quillacollo, dando a conocer una prevalencia de IRAS de $37 \%$ y una prevalencia de EDAS de $12 \%$ del total de menores de 5 años que fueron atendidos en el centro de salud de Villa Carmen, en los meses de enero a diciembre de 2017. (Ver gráfico 1)

Haciendo una comparación con otros trabajos de investigación realizados sobre la prevalencia de IRAS en el menor de 5 años, el resultado del presente estudio es similar al obtenido en un estudio del "Centro de Salud de San Benito" de la provincia de Punata, que reporto $36,4 \%$ de prevalencia de IRAS $^{8}$, el resultado del presente estudio es mayor que el reportado en el "Centro de Salud Alto San Antonio" del departamento de La Paz con una prevalencia de IRAS de 23,3\% ${ }^{9}$ y el Centro de Salud de Cuchil, en el cantón Sigsig Ecuador que reportó una prevalencia de $31,37 \%{ }^{10}$. Y es menor que el reportado en el "Centro de salud de Jadán, Azuay - Ecuador" con una prevalencia de 59,9\%, cabe mencionar que este centro de salud y su población se encuentran en una zona geográfica cerca de la cordillera de los Andes convirtiéndola en una zona endémica de infecciones respiratorias $^{11}$. En cuanto a los resultados de prevalencia de EDAS, en un estudio presentado en la Isla de San Andrés - Colombia reportó $14,2 \%$ de prevalencia ${ }^{12}$, similar al resultado del presente estudio, mientras que en el Centro de Salud de el Pangui - Ecuador se registro una prevalencia de EDAS mayor de $21,56 \%{ }^{13}$.

Realizando un análisis por mes se observa que la mayor prevalencia de IRAS es en los meses de abril, mayo, julio y la menor prevalencia es en el mes de enero. La mayor prevalencia de EDAS es en los meses de marzo, abril, junio, julio, diciembre y la menor prevalencia se registró en el mes de agosto. Se observa que los porcentajes más altos de prevalencia de IRAS coinciden con otoño e invierno y los porcentajes más bajos coinciden con verano, mientras que los porcentajes de prevalencia de EDAS se mantienen casi constantes durante todo el año. (Ver gráfico 2)

De acuerdo a la edad más afectada, en IRAS se identificó que los niños de 1 a menores de 2 años son los más afectados presentando resfrío común (133 niños enfermos) correspondiendo al 20,5\% del total de niños que enfermaron con IRAS (Ver tabla 2). Y en EDAS se identificó que los menores de 1 año son los más afectados presentando diarrea y diarrea persistente (67 niños enfermos) correspondiendo al $30 \%$ del total de niños que enfermaron con EDAS (Ver tabla 3). En un estudio realizado en el "Centro de Salud de Lacma" de Cochabamba, la mayor prevalencia de EDAS se registró en los niños menores de 1 año $^{14}$, similar al presente estudio.

El estudio realizado demuestra que la prevalencia de IRAS en la comunidad de Villa Carmen supera el $1 / 4$ del total de pacientes menores de 5 años atendidos y la prevalencia de EDAS es relativamente baja, pero si se toman en cuenta ambas patologías IRAS y EDAS la prevalencia es del 49\% (Ver 
gráfico 1) ocupando casi la mitad de la atención médica en el menor de 5 años dando a conocer la relevancia del conocimiento sobres estas patologías.

Es importante mencionar que el presente trabajo sólo incluyó a menores de 5 años y que representaría un estímulo para la realización de otros trabajos de investigación que incluyan a todos los pacientes pediátricos.

En conclusión, la prevalencia de IRAS y EDAS en la comunidad de Villa Carmen es de $37 \%$ y $12 \%$ respectivamente, datos que tienen que ser puestos a consideración por el personal de salud y las autoridades correspondientes, estas cifras nos indican que estas enfermedades pueden traer serias repercusiones en la salud de los niños si no son tratadas a tiempo. Afortunadamente en nuestro país existen programas de salud para el menor de 5 años que otorgan atención médica y medicación gratuita, contribuyendo de manera significativa en la recuperación de la salud de estos pacientes.

El resultado del presente estudio supone la posibilidad de un riesgo considerable de contraer estas enfermedades por el fácil modo de infección y desarrollo patológico en el menor de 5 años, por lo cual se recomienda la información y educación de las madres y niños respecto a medidas preventivas, haciendo hincapié en la higiene personal, principalmente en el lavado de manos con agua y jabón, de esta forma se limitaría la prevalencia de estas patologías, elevando la calidad de salud en la población.

\section{REFERENCIAS}

1.Burgoa Rivero CV, Salas Mallea AA. Conocimientos y actitudes frente a signos de alarma en infecciones respiratorias y diarreicas en niños menores de 5 años.Rev. bol. Ped [Internet]. 2008 [citado 25

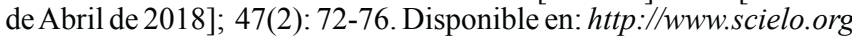
bo/scielo.php? script $=$ sci arttext\&pid $=$ S1024-06752008000200002 2.Valladares Garrido MJ. Conocimientos y prácticas de infecciones respiratorias y enfermedades diarreicas agudas en madres de una comunidad de Piura, Perú. REV CIMEL [Internet]. 2016 [citado 25 de Abril de 2018]; 21(1): 9-13. Disponible en: file:///C:/Users/Pc/ Downloads/597-1-1322-2-10-20160814.pdf

3.Turcios Ayala, GM. Conocimiento de los padres o cuidadores de los niños y niñas de dos meses a cinco años sobre los signos de alarma de las infecciones respiratorias y diarreicasagudas que buscan atención médica en la Unidad Comunitaria de Salud San Miguel. Marzo 2012. Maestría thesis, CIES UNAN-Managua [Internet]. 2012 [citado 25 de Abril de 2018]. p: 4-58. Disponible en: http://repositorio.unan.edu.ni/7228/1/t651.pdf

4.Ferreira Guerrero E, Báez Saldaña R, Trejo Valdivia B, Ferreyra Reyes L, Delgado Sánchez G, Chilián Herrera OL, et al. Infecciones respiratorias agudas en niños y signos de alarma identificados por padres y cuidadores en México. Salud pública Méx [Internet]. 2013 [citado 25 de Abril de 2018]; 55(2): S307 - S313. Disponible en: http://www.scielo.org.mx/scielo.php?pid=S0036$36342013000800030 \&$ script $=s c i$ arttext\&tlng $=e s$

5.Bell Alonso E, VinentDange Y, Formigo Montoya D, Castañeda Vargas E, BautePareta N. Modificación de conocimientos sobre la enfermedad diarreica aguda en un área de salud del municipio de II Frente. MEDISAN [Internet]. 2010 [citado 25 de Abril de 2018]; 14(8): 1068-1075. Disponible en: http://scielo.sld.cu/scielo. php? script $=$ sci_arttext\&pid $=S 1029-30192010000800004$

6.Carmona Hernández JC. Infección respiratoria aguda en relación con la contaminación atmosférica y otros factores ambientales. Archivos de Medicina (Col) [Internet]. 2009 [citado 26 de Abril de 2018]; 9(1): 69-79. Disponible en: http://www.redalyc. org/pdf/2738/273820380009.pdf

7.Guillén A. Enfermedad diarreica: un problema recurrente de salud pública. Rev. perú. med. exp. salud pública [Internet]. 2011[citado 26 de Abril de 2018]; 28(1): 07-08. Disponible en: http://www.scielo.org.pe/scielo.php?pid $=$ S172646342011000100001\&script $=$ sci_arttext

8.Chuca Tumiri A, Choque Verduguez J, Pérez Terceros MJ, Escobar Melendres E. Conducta de Infecciones Respiratorias Agudas con/ sin Neumonías en menores de 5 años del Centro de Salud "San Benito", Abril-Junio del 2010. RevCientCiencMéd [Internet]. 2011 [citado 26 de Abril de 2018]; 14(2): 12-15. Disponible en: http:// www.scielo.org.bo/pdf/rccm/v14n2/a04.pdf

9.Rojas Ortiz HA. Comportamiento de las infecciones Respiratorias agudas en menores de 5 años centro de salud Alto San Antonio gestión 2010. UMSA facmed tesis postgrado [Internet]. 2010 [citado 26 de Abril de 2018]; p: 8-77. Disponible en: http:// repositorio.umsa.bo/bitstream/handle/123456789/3671/T-PG-673. pdf? sequence $=1 \&$ is Allowed $=y$

10. Orellana Cobos DF, Urgilez Malo GJ, Larriva Villareal DK, Fajardo Morales PF. Estudio transversal: prevalencia de infecciones respiratorias agudas y su asociación con desnutrición en pacientes menores de 5 años atendidos en el centro de salud de cuchil, 2016. REVISTA MÉDICA HJCA [Internet]. 2017[citado 26 de Abril de 2018]; 9(2): 170-175. Disponible en: http://revistamedicahjca.med. ec/ojs/index.php/RevHJCA/article/view/362/342

11.Reyes Cordero A, Beltrán P, Astudillo J. Prevalencia de infecciones respiratorias agudas en pacientes menores de 5 años y su asociación con desnutrición. Jadán, enero - diciembre 2014. REVISTA MÉDICA HJCA [Internet]. 2015[citado 26 de Abril de 2018]; 7(2):100-105. Disponible en: http://revistamedicahjca.med. ec/ojs/index.php/RevHJCA/article/view/23/24

12.Duffis Forbes SA. Prevalencia de la enfermedad diarreica aguda en niños menores de cinco (5) años en San Andrés Isla: una perspectiva ambiental de los factores de riesgo. Maestría thesis Universidad Nacional de Colombia [Internet]. 2012 [citado 26 de Abril de 2018]; p: 9-78. Disponible en: http://bdigital.unal.edu. $c 0 / 46590 / 1 / 905026.2012 . p d f$

13.OROSCO SALAZAR MJ. Prevalencia de enfermedad diarreica aguda en las niñas y niños menores de cinco años atendidos en el centro de salud de el Pangui, cantón el Pangui, año 2015. Repositorio Digital Universidad Nacional de Loja [Internet]. 2016[citado 26 de Abril de 2018]; p: 2-66. Disponible en: http:// dspace.unl.edu.ec/jspui/bitstream/123456789/12354/1/Mirian\%20 Juliana\%20Orosco\%20Salazar.pdf

14.Luizaga Velasco PD, Luizaga Velasco LA, Jarro R.

Incidencia de Enfermedades Diarreicas Agudas, 2010 (Enero-

Junio), del Centro de Salud Lacma. RevCientCiencMéd

[Internet]. 2010[citado 26 de Abril de 2018]; 13(1): 9-10.

Disponible en: http://www.scielo.org.bo/scielo.php? script $=s c i$ arttext\&pid $=$ S1817-74332010000100004 